\title{
Antecedents of Two-Photon Excitation Laser Scanning Microscopy
}

\author{
BARRY R. MASTERS ${ }^{1 *}$ AND PETER T.C. SO ${ }^{2}$ \\ ${ }^{1}$ Department of Ophthalmology, University of Bern, 3010 Bern, Switzerland \\ ${ }^{2}$ Department of Mechanical Engineering, and Division of Biological Engineering, Massachusetts Institute of Technology, \\ Cambridge, Massachusetts 02139
}

\begin{abstract}
KEY WORDS nonlinear microscopy; two-photon absorption; second-harmonic generation; multiphoton
\end{abstract}

\begin{abstract}
In 1931, Maria Göppert-Mayer published her doctoral dissertation on the theory of two-photon quantum transitions (two-photon absorption and emission) in atoms. This report describes and analyzes the theoretical and experimental work on nonlinear optics, in particular two-photon excitation processes, that occurred between 1931 and the experimental implementation of two-photon excitation microscopy by the group of Webb in 1990. In addition to Maria GöppertMayer's theoretical work, the invention of the laser has a key role in the development of two-photon microscopy. Nonlinear effects were previously observed in different frequency domains (low-frequency electric and magnetic fields and magnetization), but the high electric field strength afforded by lasers was necessary to demonstrate many nonlinear effects in the optical frequency range. In 1978, the first high-resolution nonlinear microscope with depth resolution was described by the Oxford group. Sheppard and Kompfner published a study in Applied Optics describing microscopic imaging based on second-harmonic generation. In their report, they further proposed that other nonlinear optical effects, such as two-photon fluorescence, could also be applied. However, the developments in the field of nonlinear optical stalled due to a lack of a suitable laser source. This obstacle was removed with the advent of femtosecond lasers in the 1980s. In 1990, the seminal study of Denk, Strickler, and Webb on two-photon laser scanning fluorescence microscopy was published in Science. Their paper clearly demonstrated the capability of two-photon excitation microscopy for biology, and it served to convince a wide audience of scientists of the potential capability of the technique. Microsc. Res. Tech. 63:3-11, 2004. ๑ 2003 Wiley-Liss, Inc.
\end{abstract}

\section{INTRODUCTION}

The physicist Maria Göppert-Mayer developed the theory of two-photon absorption and emission processes. That was the subject of her doctoral research in Göttingen and she published her dissertation in 1931. Nonlinear effects were previously observed in other frequency domains (low-frequency electric and magnetic fields and magnetization), but the invention of the laser was necessary to demonstrate their existence at optical frequencies.

In 1978, Sheppard and Kompfner worked at Oxford and published a report in the journal Applied Optics in which they described a resonant scanning optical microscope based on second-harmonic generation (Sheppard and Kompfner, 1978). In their report, they proposed two-photon fluorescence as one of the nonlinear optical techniques that could be applied to their laser scanning microscope.

In 1990, the seminal study of Denk et al. on twophoton laser scanning fluorescence microscopy was published in the journal Science (Denk et al., 1990). This study clearly demonstrated the capability of twophoton excitation microscopy for biology, and it served to convince a wide audience of the potential capability of the technique.

The purpose of this report is to examine the antecedents to two-photon scanning laser microscopy between
1931 and 1990. The invention of a working microscope based on two-photon excitation resulted from the intersection of two technologies: laser scanning microscopy, and lasers that provide femtosecond (fs) pulses of light with high peak power. We begin with the life of Maria Göppert-Mayer. We analyze her doctoral dissertation and describe the connections with her teachers and mentors. Next is an overview of the experimental studies of optical nonlinear phenomena. The development of the scanning harmonic microscope by the Oxford group is discussed together with the physical basis of the optical sectioning capability of their microscope. This is followed by a discussion of the development of pulsed lasers. Finally, the key patent and the Science report from the Webb group are analyzed. The important advantages of multiphoton excitation microscopy are summarized and its unique capability for the threedimensional imaging of thick, highly scattering, live tissue is given.

\footnotetext{
*Correspondence: Dr. Barry R. Masters, 4001 North Ninth Street Apt. 726, Arlington, VA 22203. E-mail: brmail2001@yahoo.com

Received 10 August 2003; accepted in revised form 25 August 2003

DOI 10.1002/jemt.10418

Published online in Wiley InterScience (www.interscience.wiley.com).
} 


\section{MARIA GÖPPERT-MAYER'S SCIENTIFIC LIFE}

The scientific life of an individual provides insights to their creations and is, therefore, a valid topic in our historical discussion (Masters, 2000; McGrayne, 1996). Maria Göppert-Mayer was proud of her heritage, which was linked to seven generations of professors on her father's side of the family. When she was four, her family moved to Göttingen where her father became a Professor of Pediatrics. Her father held and expressed high expectations for his daughter; therefore, he encouraged her to strive to be more than a housewife. Early on, she decided to develop a professional career in science. Her father instilled in her a strong feeling of self-confidence and a free spirit. As she grew older, there was never doubt in her mind, or that of her parents, that she would study at the University of Göttingen.

Maria Göppert-Mayer entered the University in Göttingen to study mathematics and physics. At that time, Göttingen was the world center of these fields, especially in quantum mechanics (Van der Waerden, 1967). The Department of Mathematics hosted Richard Courant, Hermann Weyl, and Edmund Landau. Her fellow students included Max Delbrück, and Victor Weisskopf. Family friends included James Franck, Max Born, and David Hilbert.

During her Göttingen years, Maria met and interacted with the following eminent scientists: Arthur Compton, Paul Dirac, Enrico Fermi, Werner Heisenberg, John von Neumann, J. Robert Oppenheimer, Wolfgang Pauli, Linus Pauling, Leo Szilard, and Edward Teller. As a graduate student, she studied theoretical physics under Max Born at the Institute of Theoretical Physics. James Franck stands out because of his teachings and his role as her lifetime mentor. In 1931, Maria Göppert-Mayer submitted her doctoral dissertation in Göttingen on the theory of two-photon quantum transitions in atoms from the ground state to the excited state (Göppert-Mayer, 1929, 1931). The scientific content of her doctoral dissertation will be discussed more fully in the next section.

In 1930, she married Joseph Mayer, an American chemical physicist, and they moved to America, where she worked first at the Johns Hopkins University, and then at Columbia University. While at Columbia University, she met and interacted with Harold Urey, Willard Libby, Enrico Fermi, I.I. Rabi, and Jerrold Zacharias. When Edward Teller joined the University of Chicago, Maria continued her work with him. It was during her work at the Argonne National Laboratory that she developed the nuclear shell model for which she was to earn the Nobel Prize in 1963. Several years earlier, in 1955, she and Hans Jensen published a book with the title Elementary Theory of Nuclear Shell Structures. Later, in 1963, she and Jensen would share the Nobel Prize in physics for their work on nuclear shell theory. The same physics prize was also shared with Eugene Wigner. In addition to the Nobel Prize, Maria Göppert-Mayer was a member of the National Academy of Sciences, a Corresponding Member of the Akademie der Wissenschaften in Heidelberg, and received honorary degrees from Russell Sage College, Mount Holyoke College, and Smith College.

\section{MARIA GÖPPERT-MAYER'S 1931 DOCTORAL DISSERTATION}

In 1931, Maria Göppert-Mayer submitted her doctoral dissertation in Göttingen on the theory of twophoton quantum transitions in atoms (Göppert-Mayer, 1931). However, she published a preliminary report on her theory two years earlier (Göppert-Mayer, 1929). In this one-page report, which she signed Maria Göppert, she formulated energy state diagrams for both twophoton emission and two-photon absorption processes. She indicated the presence of virtual states. She also concluded that the probability for the two-photon absorption process is proportional to the square of the light intensity.

It is a delight to read her 1931 report, which is a model of clarity and could be part of a textbook on quantum mechanics. She cited several references in her dissertation: the work of Dirac on the matrix formulation of quantum mechanics, the work of Kramers and Heisenberg on the quantum mechanical formulation of the problem of single quantum processes in absorption and emission of light, the work of Franck on the intensity of spectral lines from electronic transitions in an atom, and the work of Born on the theory of ionization processes. At the end of her dissertation, she thanks Professor Born for friendly help and advice, and Victor Weisskopf, a fellow graduate student, for discussion and help. Finally, she wrote "John Hopkins University" (that may be a misprint of Johns Hopkins University) at the end of her dissertation as the place where she wrote her paper. Maria Göppert-Mayer's complete 1931 dissertation is reprinted in Selected Papers on Multiphoton Excitation Microscopy (Masters, 2003).

Maria Göppert-Mayer followed the technique of Dirac for the use of perturbation theory to solve the quantum mechanical equations for the processes of absorption, emission, and dispersion of light in single photon-atom interactions. Dirac demonstrated the equivalence of the Schrödinger and the Heisenberg formulations of wave mechanics among his many scientific contributions (Dirac, 1958). In her doctoral thesis, Maria Göppert-Mayer extended the quantum mechanical treatment of Dirac to electronic processes that involve two annihilation (or two creation) operators in order to describe both two-photon absorption and emission processes. The transition probability of a twophoton electronic process was derived by Maria Göppert-Mayer using second-order, time-dependent, perturbation theory (Peebles, 1992). Her derivation clearly states that the probability of a two-photon absorption process is quadratically related to the excitation light intensity. Her theory applied not only to two-photon absorption, two-photon emission, but also to Stokes and anti-Stokes Raman effects.

An important aspect of Maria Göppert-Mayer's thesis is that the process of two-photon absorption involves the interaction of two photons and an atom via an intermediate virtual state. This interaction must occur within the lifetime of this virtual state. What is a virtual state? It can be described as a superposition of states and not a true eigenstate of the atom. Therefore, the probability of the two-photon transition has contributions from all of the intermediate states. The first 
photon induces the transition from the ground state to the virtual state, and the second photon induces the transition from the virtual state to the excited state. Both photons interact to induce the transition from the ground state to the excited state. Since the probability of the two-photon absorption processes is very low, it is necessary to use a high-intensity light in order to achieve a measurable effect.

Out of respect for the work of Maria Göppert-Mayer, the units of two-photon absorption cross-section are measured in GM (Göppert-Mayer) units. One GM unit is equal to $10^{-50} \mathrm{~cm}^{4} \mathrm{~s} /$ photon.

Since the 1931 Göttingen dissertation is written in German, it is helpful to follow it together with the derivation of Boyd for those readers who cannot read German. Boyd used time-dependent perturbation techniques to solve the quantum mechanical equations and derived the probabilities and cross-sections for singlephoton absorption, two-photon absorption, and multiphoton absorption (Boyd, 2003).

\section{NONLINEAR OPTICAL PROCESSES AND MICROSCOPY}

What are nonlinear optical processes? They occur when "the response of a material system to an applied optical field depends in a nonlinear manner upon the strength of the optical field" (Boyd, 2003). An early example of a nonlinear optical effect is optical pumping. Low-power atomic lamps were used in experiments on atomic systems. The resonant excitation of optical pumping changes the optical properties of the medium, and the resonant enhancement makes possible the detection of this effect even with weak illumination (Shen, 1984).

In general, a bright light source is necessary to detect nonlinear optical effects. For example, in second-harmonic generation, the intensity of the light generated at the second-harmonic frequency increases as the square of the intensity of the incident laser light. Second harmonic generation is the first nonlinear optical effect ever observed in which a coherent input generates a coherent output (Shen, 1984). For a two-photon absorption process, the intensity of the fluorescence generated in this process increases as the square of the intensity of the incident laser light.

The investigation of these phenomena becomes possible with the development of the laser that provides an intense source of monochromatic, coherent light (Maiman, 1960). When the medium has a quadratic relationship between the polarization density and the electric field, there are second order nonlinear optical phenomena: second-harmonic generation with the frequency doubling of monochromatic light, frequency conversion with the sum or difference of two monochromatic waves giving the frequency of a third wave. Similarly, a third-order relationship between the polarization density of the medium and the electric field gives rise to third-harmonic generation, self-focusing, optical amplification, and optical phase conjugation (Bloembergen, 1965, 1996a,b; Boyd, 2003; Shen, 1984). The Quantum Theory of Radiation is recommended as a source of general background to radiative processes (Heitler, 1954).

Experimentally, stimulated Raman scattering (a two-photon process) was discovered in the early 1960 s
(Woodbury and Ng, 1962). Woodbury and Ng were investigating Q-switching in a ruby laser with a Kerr cell that contained nitrobenzene. They discovered that there was a component of the laser output that was at $1,345 \mathrm{~cm}^{-1}$ and it was downshifted from the laser frequency. Further analysis indicated that this frequency shift was identical to the frequency of vibration of the strongest Raman mode of the nitrobenzene molecule (Boyd, 2003). Raman scattering can be viewed as a two-photon process in which one photon is absorbed and one photon is emitted when the molecules undergo the transition from the initial state to the final state. The terms Stokes and anti-Stokes refers to a shift to lower frequencies, or a shift to higher frequencies of the scattered photon with respect to the frequency of the photons in the incident light. The Stokes components are usually orders of magnitude more intense than the anti-Stokes components.

Nonlinear optical spectroscopy preceded the development of nonlinear microscopy. The group of Franken made the first observation of second-harmonic generation (SHG) in a quartz crystal irradiated with a ruby laser (Franken et al., 1961). The authors stated that the development of pulsed ruby optical masers that output monochromatic light $(6,943 \mathrm{~A})$ can be focused and exhibit electric fields of $10^{+5}$ volts $/ \mathrm{cm}$. When this light is focused onto a quartz crystal, the second harmonic signal at $3,472 \mathrm{~A}$ is produced. It is critical that the crystal lacks a center of inversion and is transparent to both the incident light at 6,943 $\mathrm{A}$ and the second harmonic light at $3,472 \mathrm{~A}$. The authors were careful to present several controls: the light at 3,472 A disappears when the quartz specimen is removed or is replaced by glass, and the light at 3,472 A shows the expected dependence on polarization and crystal orientation. Furthermore, they predicted that isotropic materials such as glass could also show second-harmonic generation in the presence of a strong bias electric field.

Bloembergen, in his report "Nonlinear optics: past, present, and future" (2000), relates an interesting historical aspect of scientific publication. Franken et al. (1961) passed a ruby laser pulse through a quartz crystal. They used a monochromator and a spectrographic plate to detect ultraviolet light from the experiment. However, since the spot of the second-harmonic was so weak on the spectrographic plate, the editorial office of Physical Review removed the spots from the figure prior to publication. An erratum was never published!

Another interesting nonlinear phenomenon is optical mixing (Bloembergen, 1986; Boyd, 2003). In 1962, Bloembergen and coworkers published a classic paper on the theoretical basis of optical mixing (Armstrong et al., 1962), while Franken et al. (1961) showed that small quadratic terms in the optical polarizability of piezoelectric materials made possible the production of the optical second-harmonic when irradiated with intense red light from a ruby laser. These same terms would permit the mixing of light from two lasers at different frequencies. It was shown that two ruby laser beams of different frequencies that are coincident simultaneously on a crystal will produce the sum frequency in the near ultraviolet (Bass et al., 1962). The two frequencies were obtained from a ruby laser oper- 
ating at liquid nitrogen temperature, and a ruby laser operating at room temperature.

Kaiser and Garrett, who worked at Bell Telephone Laboratories, Murray Hill, New Jersey, made the first experimental observation of two-photon excitation fluorescence in 1961. Their report was published only a few weeks after the publication of Franken's paper and they also used a ruby optical maser for their studies (Kaiser and Garrett, 1961). They generated blue fluorescent light around a wavelength of 4,250 A by illuminating crystals of $\mathrm{CaF}_{2}: \mathrm{Eu}^{2+}$ with red light at 6,943 A from a ruby optical maser. The authors state that the appearance of fluorescence indicated that the $\mathrm{Eu}^{+2}$ was in the excited state, and that the excited state was excited by a two-photon process. The authors show a log-log plot of fluorescent intensity vs. incident intensity with a slope of two. They state that the probability of an atom being excited by two photons, the sum of whose energies corresponds to the excited state of the atom, was first treated in 1931 by Maria Göppert-Mayer. Maria Göppert-Mayer's theoretical paper was listed in the references. The authors calculated the efficiency for the two-photon excitation process under their experimental condition as $10^{-7}$. The efficiency was calculated as the ratio of the number of fluorescence photons generated and the number of incident photons. The Kaiser and Garrett paper was published 30 years after two-photon absorption at optical frequencies was predicted by Maria GöppertMayer.

Higher-order multi-photon processes, for example, three-photon excitation fluorescence, were observed in the following years. Three-photon absorption processes were first reported in naphthalene crystals (Singh and Bradley, 1964). In the first report of a three-photon electronic transition, they used a ruby laser with a crystal of naphthalene and reported on the observed ultraviolet fluorescence. The intensity of the fluorescence signal increased as the third power of the laser intensity, which is expected for this nonlinear process. Rentzepis and coworkers observed and photographed three-photon excited fluorescence in organic dye molecules (Rentzepis et al., 1970). Other spectroscopic studies using multiphoton fluorescence processes followed (Birge, 1983, 1986; Friedrich and McClain, 1980; Friedrich, 1982; McClain, 1971; McClain and Harris, 1977).

An advantage of multiphoton absorption is that the selection rules for electronic transitions from singlephoton interactions are not valid (Hunt, 2000; Shen, 1984). The selection rules for electronic transitions can be summarized as follows: two-photon absorption is an even-parity transition, and single-photon absorption is an odd-parity transition between the ground and excited electronic states. A single-photon transition can occur between electron states with opposite parity. A two-photon electronic transition between the ground state and the excited state can couple states wit the same parity. Two-photon absorption processes can excite higher energy states. In multiphoton spectroscopy, it is possible to study Rydberg states that contain high quantum numbers. Multiphoton disassociations of molecules can explore molecular spectroscopy of high-energy states.

The next step in the application of nonlinear optics was the development of a nonlinear optical microscope for the examination of the microscopic structure of polycrystalline ZnSe (Hellwarth and Christensen, 1974). They used a conventional harmonic microscope in which the entire specimen was wide-field illuminated with the laser beam, and the image of polycrystalline ZnSe was formed by imaging the emitted harmonic radiation. Their second-harmonic microscope used a repetitively Q-switched Nd:YAG laser giving $10^{+3}$ pulses per second at $1.06 \mu$, each of $10^{-4} \mathrm{~J}$ energy and $2 \times 10^{-7}$ second duration. The green second-harmonic was viewed by a microscope or on a Polaroid film. Their nonlinear second-harmonic generation microscope was used to observe single-crystal platelets that were not visible in an ordinary polarizing light microscope. Hellwarth and Christensen developed the theory for the second-harmonic generation in polycrystalline ZnSe. Their analysis indicated that phase-matched second harmonic generation does not exist in a singlecrystal ZnSe since it is isotropic; however, spatial variation of the second harmonic nonlinear susceptibility (a third rank tensor) in the polycrystalline ZnSe can create the necessary phase-matching that creates the second-harmonic light emission. The next major advance took place at Oxford University.

\section{SCANNING HARMONIC OPTICAL MICROSCOPE AT OXFORD UNIVERSITY}

In 1977, the Oxford group of Sheppard, Kompfner, Gannaway, Choudhury, and Wilson first suggested and then demonstrated how a nonlinear optical phenomenon would be incorporated into their high-resolution, three-dimensional resolved scanning laser microscope (Sheppard and Kompfner, 1978).

The principle of nonlinear scanning microscopy is simply explained in the Theory and Practice of Scanning Optical Microscopy (Wilson and Sheppard, 1984). In a conventional light microscope, the object is illuminated with full-field illumination from an extended source through a condenser lens and the illuminated patch of the specimen is imaged by the objective lens into the image plane, and then it is viewed through an eyepiece. The resolution is due primarily to the objective lens, and the optical aberrations due to the condenser are less important. In such a conventional microscope, the source of the contrast is the differences in the absorption coefficients and the optical thickness of the specimen. If a high-intensity beam of light impinged on a specimen, the specimen would behave in a nonlinear manner and higher optical harmonics would be produced. This nonlinear harmonic generation would be a function of the molecular structure of the specimen. All materials possess third-order nonlinear susceptibility together with higher order terms, $5,7,9 . \ldots$. Second-order nonlinear susceptibility exists in crystals that have noncentrosymmetric geometry; for example, crystals such as $\mathrm{LiNbO}_{3}$, and some biological specimens.

The Oxford group suggested developing nonlinear scanning optical microscopes where the excitation light is focused to a small volume and an image is generated based on raster scanning of either the light or the specimen. Since the excitation light is focused to the diffraction limit, the electric field strength at the focal volume is significantly greater than the wide-field illumination geometry of previous designs. The signifi- 
cantly higher field strength allows a vastly more efficient generation of optical harmonics since the nth order harmonic signal is generated in proportion to the nth power of the fundamental intensity. For example, second-harmonic generation depends quadratically on the incident light intensity.

The Oxford group also pointed out an important consequence of the nonlinear dependence of the emission signal with the excitation light: super resolution. If the fundamental radiation had a Gaussian distribution, the harmonic radiation will also have a Gaussian distribution, but the radius will be only $1 / \sqrt{ } n$ of that of the fundamental beam (Wilson and Sheppard, 1984). Axially, they further noted that this system will have depth discrimination because the intensity point spread function of this microscope is a quadratic function of the intensity transfer function of the objective similar to an incoherent confocal microscope.

Wilson and Sheppard further suggested that a laser microscope could be used to investigate the nonlinear optical properties of a specimen based on variations in the specimen's second-order susceptibility. In addition to harmonic generation, they further realized that other nonlinear effects such as Raman scattering, sum frequency generation, or two-photon fluorescence could be used to study the energy levels and hence the chemical structure of the specimen (Wilson and Sheppard, 1984).

Sheppard and his coworkers made the first conference presentation of a scanning second-harmonic generation microscopy in 1977 (Sheppard et al., 1977). They showed a schematic for a scanning harmonic microscope. They noted that the second-harmonic is formed in the forward direction. The specimen was scanned relative to the focused laser beam and the focused beam produced optical second harmonics in the specimen itself. They proposed that a second-harmonic microscope could be used to image biological structures with a very high contrast. They stated the temperature rise must be small in biological samples. They calculated that $1 \mathrm{~W}$ of incident light may produce $10^{-10} \mathrm{~W}$ of second-harmonic light. To keep the specimen temperature rise low, the laser beam and not the sample must be scanned. The authors built a specimen scanning second-harmonic microscope based on a 1-W CW neodymium YAG laser; while it was unsuitable for biological specimens, it was used to image crystals. They further illustrated the optical sectioning capability of their harmonic generation microscope by imaging various planes within a thin crystal.

Gannaway and Sheppard (1978) contributed the first full journal report on scanning second-harmonic generation microscopy, with a discussion of the advantages of a pulsed beam and heating effects. This is an extended version of their 1977 conference communication. They noted that second-harmonics are generated only by molecules that are noncentrosymmetiric and contrast in the microscope is a function of molecular structure of the specimen and its orientation with respect to the laser beam. The authors stated their ultimate aim is to have a second harmonic microscope to examine biological specimens. They pointed out the advantage of the scanning technique in nonlinear microscopy since a much lower laser power is necessary to achieve a given power density in the specimen. They also pointed out the advantage of using pulsed lasers to enhance the conversion of the fundamental to harmonic power in a scanning optical microscope. Their scanning laser microscope was a high-resolution system as compared to the previous microscope of Hellwarth and Christensen, which has resolution in the range of $20-200 \mu$.

Later, the Oxford group proposed several microscope configurations for the enhancement of nonlinear interactions in the scanning optical microscope such as placing the specimen in a resonant cavity, and using beam pulsing (pulsed lasers) to improve the efficiency of conversion of fundamental to harmonic power (Sheppard and Kompfner, 1978). In the same report, they proposed various types of nonlinear interactions for use in the scanning optical microscope: the generation of sum grequencies, Raman scattering, and two-photon fluorescence.

Thus, the development of the scanning laser harmonic microscope solved many of the problems of nonlinear microscopy: the advantages of diffraction limited laser scanning, the analysis and mitigation of laser heating effects and subsequent thermal damage by laser scanning, the confinement of the second-harmonic generation to the focal volume of the microscope objective, and the origin of the optical sectioning effects based the physics of the process. The Oxford group also suggested that the efficiency of conversion of the fundamental to harmonic power would be increased by pulsing the laser (Masters, 1996).

\section{ROLE OF LASERS IN THE DEVELOPMENT OF MULTIPHOTON EXCITATION MICROSCOPY}

The invention of a new instrument can be an important antecedent in scientific discovery. This is obvious in the field of nonlinear optics. Following the development of the first laser in 1960 by Maiman, there were many discoveries: such as the second-harmonic generation of light (Franken et al., 1961) and the two-photon excited fluorescence (Kaiser and Garrett, 1961). The ruby laser was also used to demonstrate third-harmonic generation and anti-Stokes frequency mixing.

In 1961, the technique of Q-switching was used to obtain shorter laser pulses with higher peak intensities (Siegman,1986). Laser pulses of picosecond duration were obtained by passive mode locking with a saturable dye cell. In fact, picosecond laser pulses have been used to induce two-photon absorption in organic molecules (Bradley et al., 1972).

In the $1970 \mathrm{~s}$, it was possible to obtain femtosecond ( $1 \mathrm{fs}$ is equal to $10^{-15} \mathrm{~s}$ ) laser pulses based on a combination of saturable gain in a dye laser medium and a saturable dye absorber in a ring laser cavity mode together with compensation of the dispersion in group velocity (Schäfer and Müller, 1971). The early images from the Webb group using multiphoton excitation microscopy were obtained with a colliding-pulse $80-\mathrm{MHz}$ mode-locked dye laser that generated 100 -fs pulses at $630 \mathrm{~nm}$ (Valdemanis and Fork, 1986). The disadvantage of this laser is rather limited tunability and stability.

In the $1990 \mathrm{~s}$, the development of the Ti-sapphire femtosecond laser resulted in a laser source that was optimal for two-photon excitation microscopy (Spence 
et al., 1991). The Ti:sapphire lasers are self-modelocked and use the Kerr lens effect to generate modelocked pulses with output pulse widths in the fs range. This is due to the electric field generated by a strongly focused Gaussian laser beam causing an inhomogeneous change in the refractive index of the Ti:sapphire crystal. This change creates a weak lens in the crystal that results in a higher gain for mode-locked laser pulses than for continuous wave (cw) pulses. Titaniumsapphire lasers offer a broad tunability, which spans the range of 700-1,100 $\mathrm{nm}$ (Spence et al., 1991). The titanium-sapphire laser could be pumped with either an argon-ion laser or a solid state semiconductor laser. The pulses are generated by the self mode-locking Kerr effect in the titanium-sapphire lasing rod. An alternative laser source with a large band of spectral coverage can be obtained from a titanium-sapphire laser that is coupled to a regenerative amplifier (Salin et al., 1991).

A critical component of a multiphoton microscope is the laser light source (Masters et al., 1999). The fluorescence excitation rate is sub-optimal for scanning microscopy applications unless femtosecond (fs) or picosecond (ps) pulsed lasers are used although imaging using continuous wave lasers has been demonstrated. The choice between femtosecond and picosecond light sources for multiphoton excitation remains controversial. As discussed, multiphoton excitation can be achieved with both types of lasers. In order to generate the same level of fluorescence signal, the picosecond laser systems will need a significantly high average input light intensity while the femtosecond laser systems has a much higher peak power. For a pulsed laser, the following parameters are important. The energy per pulse is the average power divided by the pulse rate. The peak power is approximately the energy per pulse divided by the pulse width. The duty cycle of the laser is given by the pulse width multiplied by the pulse rate.

Today, several ultrafast laser sources are available for multiphoton excitation microscopy. The important considerations in laser selection for multiphoton excitation microscopy include: peak power, pulse width, repetition rate, range of wavelength tunability, cooling requirement, power requirement, and cost. The range of tunability should cover the multiphoton spectra of the fluorophores of interest.

Titanium-sapphire laser systems are most commonly used for multiphoton excitation microscopy. These systems provide high average power $(1-2 \mathrm{~W})$, high repetition rate (80-100 MHz), and short pulse width (80-150 fs). The Titanium-sapphire laser provides a wide tuning range from below $700 \mathrm{~nm}$ to above $1,000 \mathrm{~nm}$. Titaniumsapphire lasers require pump lasers. The older systems use Argon-ion lasers. A recent development was the use of all solid-state pump lasers accomplished by using an array of semiconductor lasers exciting $\mathrm{Nd}: \mathrm{YVO}_{4}$ crystals (Lamb et al. 1994; Spence et al, 1991). This eliminated the need for the less efficient gas lasers to pump the Ti-sapphire laser.

Turnkey Ti-sapphire laser systems have been developed that are compact, and have good tunability. Other single wavelength solid state systems such as diode pumped Nd:YLF lasers, diode pumped Erbium doped fiber laser systems, and the Cr:LiSAF laser described by French et al. (1993) have been developed. They trade off system versatility with cost.

The early work by Hellwarth and Christensen in 1974 on their second-harmonic microscope used a repetitively Q-switched Nd-YAG laser. The early implementations of nonlinear optical microscopy at Oxford used a continuous wave laser scanning microscope to image second harmonic generation in crystals (Gannaway and Sheppard, 1978; Sheppard and Kompfner, 1978; Wilson and Sheppard, 1979). It was the development of the mode-locked laser that generated femtosecond laser pulses at a repetition rate of $80-100 \mathrm{MHz}$ that provided the intense light source for the next step in the development of the multiphoton excitation microscope.

\section{THE DENK, STRICKLER, AND WEBB SCIENCE REPORT (1990) AND THEIR PATENT (1991)}

It was the seminal work of Denk and colleagues, published in Science in 1990, that launched a new revolution in nonlinear optical microscopy (Denk et al., 1990). The patent application was filed on November 14, 1989. On July 23, 1991 a United States Patent on "Two-photon laser microscopy" was assigned to the Cornell Research Foundation, Inc., Ithaca, New York (Denk et al., 1991). The patent lists Winfried Denk, James Strickler, and Watt W. Webb as the inventors. By integrating a laser scanning microscope (scanning mirrors, photomultiplier tube detection system) and a mode-locked laser that generates pulses of near-infrared light, they succeeded in demonstrating a new type of fluorescent microscope based on two-photon excitation of molecules. The pulses of red or near-infrared light $(700 \mathrm{~nm})$ were less than $100 \mathrm{fs}$ in duration and the laser repetition rate was about $100 \mathrm{MHz}$. The patent states that "focused subpicosecond pulses of laser light" are used (Denk et al., 1991). These pulses have sufficiently high peak power to achieve two-photon excitation at reasonable rates at an average power less than $25 \mathrm{~mW}$, which is tolerable to biological samples.

The high-intensity, short pulses of near-infrared light causes significant multiphoton excitations; however, the relative transparency of cell and tissue for infrared radiation and the lower average power minimizes photo-damage. The benefits of two-photon excitation microscopy include: improved background discrimination, reduced photobleaching of the fluorophores, and minimal photodamage to biological specimens. The inventors proposed the application of two-photon excitation microscopy for optical sectioning three-dimensional microscopy and for the uncaging of molecules inside of cells and tissues.

It is interesting to note the publications cited in the patent and their publication dates. Pages 8 and 9 of the Wilson and Sheppard book Theory and Practice of Scanning Optical Microscopy are cited (Wilson and Sheppard, 1984). The two key points from these pages are (1) the discussion of the physical basis of the optical sectioning in a nonlinear second harmonic microscope; the quadratic nature of the harmonic generation confines the effect to the focal region, and the concomitant sharpening of the beam in the lateral direction; and (2) the suggestion that other nonlinear optical effects, including Raman scattering or two-photon fluorescence, 
can be used for scanning laser microscopy. Many of the reports and patents that are relevant to multiphoton excitation microscopy have been reprinted in a book (Masters, 2003).

The patent can be summarized by the following sentence from the Abstract, "A laser scanning microscope produces molecular excitation in a target material by [the] simultaneous adsorption of two photons to thereby provide intrinsic three-dimensional resolution." (Addition). The patent also states, "the focused pulses also provide three-dimensional spatially resolved photochemistry which is particularly useful in photolytic release of caged effector molecules."

The patent gives the light source as: "strongly focused subpicosecond pulses of laser light." The strong focusing occurs only in the focal region of the microscope objective and is similar to the origin of the optical sectioning in the second harmonic microscope described by Wilson and Sheppard (Wilson and Sheppard, 1979). The laser scanning microscope described in the patent is similar to instruments described by others in prior art (the patent cited 14 previous laser scanning instruments). The statement regarding subpicosecond opens a window for picosecond lasers to be used for two-photon laser microscopy without infringing on the Denk et al. 1991 patent. As early as 1972, picosecond lasers were used for two-photon absorption studies and measurement of absorption cross-sections (Bradley et al., 1972).

In the section of the patent labeled "Background of the invention," the authors review the various types of confocal microscopes, their light sources and scanning mechanisms. The authors clearly state the limitations of confocal microscopy as applied to fluorescent molecules that are excited in the ultraviolet: (1) the lack of suitable microscope objectives for the ultraviolet that are chromatically corrected and transparent for both the absorption and emission wavelengths, (2) photodamage to living cells by the ultraviolet light, and (3) the problem of photobleaching of the fluorophores.

In the section labeled "Summary of the invention," the authors propose that their invention overcomes these difficulties. The authors state that the two-photon excitation is made possible by (1) a very high, local, instantaneous intensity provided by the tight focusing of the laser scanning microscope in which the laser beam is focused to a diffraction-limited waist of less than $1 \mu \mathrm{m}$, and (2) the temporal compression of the pulsed laser. This process yields improved background discrimination, reduced photobleaching, and minimizes the photodamage to living specimens. The physics of the process is clearly described by the authors in the following sentence from their patent: "only in the region of the focal point on the object plane at the waist formed by the converging and diverging cones is the intensity sufficiently high to produce two-photon absorption in the specimen fluorophore, and this intensity dependence enables long wavelength light to provide the effect of short wavelength excitation only in the small local volume of the specimen surrounding the focal point."

The patent further provides a formula for the number of photon absorbed per molecules of fluorophore per pulse as a function of pulse duration, repetition rate, average power of the incident laser, the numerical ap- erture of the focusing lens and the photon absorption cross section. In a two-photon excitation process, the number of photons absorbed per molecule of fluorophore per pulse scales with the average incident laser power squared. This is the source of the experimental verification of two-photon excitation processes; on a log-log plot of detected intensity vs. laser power the slope of the plot is 2 . The authors also state that the two-photon excitation fluorescence can be increased by increasing the pulse repetition frequency until saturation of the excited state is achieved.

Another key feature of the patent is the description of a non-descanned detection of the fluorescence intensity derived from the two-photon absorption process. Since the fluorescence signal depends on the square of the excitation intensity, there is an optical sectioning effect through the specimen even in the absence of a pinhole being used as a spatial filter in front of the detector. Therefore, the detector can be a large area detector such as a photomultiplier tube. This avoids many of the problems associated with conventional laser scanning confocal microscopes. With the publication of the Science report and the patent from the Webb group in 1991, the reality of two-photon excitation microscopy began.

Webb and coworkers further stated that the microscope can be operated in sum or difference frequency mode. It is not necessary that the two photons that are absorbed in a two-photon excitation process be of the same wavelength. In the sum frequency case, an excitation transition requiring energy hc/ $\lambda_{a b}$ can be achieved using lasers with wavelengths, $\lambda_{\mathrm{a}}$ and $\lambda_{\mathrm{b}}$, based on the following conservative equation:

$$
1 / \lambda_{\mathrm{ab}}=1 / \lambda_{\mathrm{a}}+1 / \lambda_{\mathrm{b}}
$$

where $h$ and c are Planck's constant and the speed of light (Webb patent, 1991).

\section{COMPARISON OF MULTIPHOTON EXCITATION MICROSCOPY AND CONFOCAL MICROSCOPY}

The advantages of these nonlinear microscopes include improved spatial and temporal resolution without the use of pinholes or slits for spatial filtering. Since the optical sectioning capability of the two-photon excitation microscope derives from the physics of the excitation process, there is no requirement for a spatial filter in front of the detector as in confocal microscopy (Corle and Kino, 1996). Two-photon microscopy further allows deeper penetration into thick, highly scattering tissues, and confines photobeaching and photodamage to the focal volume.

The main limitations of two-photon excitation microscopy are (1) multiphoton excitation microscopy is only suitable for fluorescent imaging; reflected light imaging is not possible, and (2) the technique is not suitable for imaging highly pigmented cells and tissues that absorb near-infrared light.

One can further compare these two techniques based on spatial resolution. Just after the Denk et al. Science report was published, the image formation properties in two-photon fluorescence microscopy was compared with confocal microscopy (Sheppard and $\mathrm{Gu}, 1990$ ). 
These authors compared both transverse and axial resolution for both types of microscopy and concluded that confocal microscopy has a higher spatial resolution than multiphoton excitation microscopy. Further analysis and comparison of the transfer functions for a one-photon and a two-photon process are in agreement with previous analyses (Gu, 1996; Gu and Sheppard, 1995).

Experimental comparisons of these two types of microscopies have been performed including a study in which we imaged in vivo human skin with both multiphoton excitation microscopy and tandem scanning reflected light confocal microscopy (Masters and So, 1999; Masters et al., 1997). These studies demonstrate that multiphoton excitation microscopy has the capacity to image deeper within highly scattering tissues such as in vivo human skin. For observation of the dermis, the ability of the multiphoton excitation microscope to image the elastin fibers and the collagen fibers within the tissue is a significant advantage.

\section{DISCUSSION}

With the publication in 1990 of the Science report from the Webb group, the biological community was convinced of the capability of multiphoton excitation microscopy for the imaging of live cells, tissues, embryos, and organisms. The application of multiphoton excitation microscopy has resulted in many important experimental studies in biology and medicine. A few of these studies are described below by way of illustration.

In the area of cellular oxidative metabolism, twophoton excitation laser scanning microscopy was used to investigate alterations in the $\mathrm{NAD}(\mathrm{P}) \mathrm{H}$ levels in the basal cells of the in situ cornea (Piston et al., 1995). The two-photon excitation microscopy technique resulted in improved sensitivity and spatial resolution as compared to previous techniques.

The field of embryology and developmental biology is benefiting from the studies that use multiphoton excitation microscopy to investigate the embryological development with less photodamage and more spatial resolution than that of older techniques. A good illustration of this application is a study on the orientation of the first cleavage in sea urchin embryo (Summers et al., 1996). A major challenge to the study of developing embryos is to achieve long-term microscopic observation without photo-induced damage. A study that demonstrated this with mammalian embryos showed that multiphoton excitation microscopy, but not confocal microscopy, could be used to monitor embryos at frequent intervals over a 24-hour period (Squirrel et al., 1999).

Neurobiology is another field that has actively applied multiphoton excitation microscopy. An important area of research is that of neural plasticity; how does the neural system grow, change, develop, and respond to sensory experience. An interesting application of multiphoton excitation microscopy is a study of longterm in vivo imaging of synaptic plasticity in adult cortex (Trachtenberg et al., 2002). These investigators found that sensory experience drives the formation and the elimination of synapses and that these alterations may underlie adaptive remodeling of neural circuits.

The publication in 1931 of Maria Göppert-Mayer's doctoral dissertation on the theory of two-photon quan- tum transitions (two-photon absorption and emission) in atoms was the theoretical foundation of the chain of scientific and technology development that leads to multiphoton microscopy. The publications in the last decade in biology and medicine that are based on the multiphoton excitation microscope demonstrates its capabilities well, and they are a fitting tribute to the doctoral work of Maria Göppert-Mayer, and to all the other scientists who contribute to the field of nonlinear optical microscopy (Masters and So, 2004).

\section{ACKNOWLEDGMENTS}

B.R.M. is thankful for the support from the 1999 Alfred Vogt-Prize for Ophthalmology (the highest award in Switzerland for scientific research in ophthalmology) from the Alfred Vogt-Stiftung zur Förderung der Augenheilkunde Zürich, that he shared with Professor M. Böhnke, for their work "Confocal Microscopy of the Cornea." We thank Katie Lagoni-Masters for obtaining a copy of the 1929 thesis of Maria Göppert from the library of the University of Göttingen.

\section{REFERENCES}

Armstrong JA, Bloembergen N, Ducuing J, Pershan PS. 1962. Interactions between light waves in a nonlinear dielectric. Phys Rev 127:1918-1939.

Bass M, Franken PA, Hill AE, Peters CW, Weinreich G. 1962. Optical mixing. Phys Rev Lett 8:18.

Boyd RW. 2003. Nonlinear optics, 2nd ed. New York: Academic Press. Birge RR. 1983. One-photon and two-photon excitation spectroscopy. In: Kliger DS, editor. Ultrasensitive laser spectroscopy. New York: Academic Press. p 109-174.

Birge RR. 1986. Two-photon spectroscopy of protein-bound fluorophores. Acc Chem Res 19:138-146.

Bloembergen N. 1965. Nonlinear optics. New York: Benjamin.

Bloembergen N. 1996a. Nonlinear optics: a historical perspective. In: Encounters in nonlinear optics, selected papers of Nicolaas Bloembergen. Singapore: World Scientific. p 122-133.

Bloembergen N. 1996b. Encounters in nonlinear optics, selected papers of Nicolaas Bloembergen. Singapore: World Scientific.

Bloembergen N. 2000. Nonlinear optics: past, present, and future

IEEE J Select Top Quant Electron 6:876-880.

Bradley DJ, Hutchinson MHR, Koetser H. 1972. Interactions of picosecond laser pulses with organic molecules. Two-photon absorption cross-sections. Proc R Soc Lond A. 329:105-119.

Corle TR, Kino GS. 1996. Confocal scanning optical microscopy and related imaging systems. San Diego: Academic Press.

Denk W, Strickler JH, Webb WW. 1990. Two-photon laser scanning fluorescence microscopy, Science 248:73-76.

Denk WJ, Strickler JP, Webb WW. 1991. Two-photon laser microscopy. United States Patent, 5,034,613, July 23, 1991.

Diaspro A. 2002. Confocal and two-photon microscopy: foundations, applications, and advances. New York: Wiley-Liss.

Dirac PAM. 1958. The principles of quantum mechanics, 4th ed. London: Oxford University Press.

Franken PA, Hill AE, Peters CW, Weinreich G. 1961. Generation of optical harmonics. Phys Rev Lett 7:118-119.

French PMW, Mellish R, Taylor JR, Delfyett PJ, Florez LT. 1993. All solid-state diode-pumped modelocked Cr:LiSAF laser. Electron Lett 29:1263-1264.

Friedrich DM. 1982. Two-photon molecular spectroscopy. J Chem Educ 59:472-483.

Friedrich DM, McClain WM. 1980. Two-photon molecular electronic spectroscopy. Annu Rev Phys Chem 31:559-577.

Gannaway JN, Sheppard CJR. 1978. Second harmonic imaging in the scanning optical microscope. Optics Quant Electron 10:435-439.

Göppert-Mayer, M. 1929. Über die Wahrscheinlichkeit des Zusamenswirkens zweier Lichtquanten in einem Elementarakt. Naturwissenschaften 17:932.

Göppert-Mayer, M. 1931. Über Elementarakte mit zwei Quantensprüngen. Ann Phys (Leipzig) 9:273-294.

$\mathrm{Gu}$ M. 1996. Principles of three-dimensional imaging in confocal microscopes. Singapore: World Scientific. p 164-173.

Gu M, Sheppard CJR. 1995. Comparison of three-dimensional imaging properties between 2-photon and single-photon fluorescence microscopy. J Microsc 177:128-137. 
Heitler W. 1954. The quantum theory of radiation. London: Oxford University Press. Reprinted in 1983 by Dover Publications, New York.

Hellwarth R, Christensen P. 1974. Nonlinear optical microscope examination of structures in polycrystalline ZnSe. Opt Commun 12: $318-322$.

Hunt JH. 2000. Selected papers on nonlinear optical spectroscopy, MS 160. SPIE. Bellingham, WA: The International Society for Optical Engineering.

Kaiser W, Garrett CGB. 1961. Two-photon excitation in $\mathrm{CaF}_{2}: \mathrm{Eu}^{2+}$. Phys Rev Lett 7:229-231.

Lamb K, Spence D, Hong J, Yelland C, Sibbert W. 1994.. All solid state self mode locked Ti:sapphire laser. Opt Lett 19:1864-1866.

Maiman TH. 1960. Stimulated optical radiation in ruby. Nature 187: 493-494.

Masters BR. 1996. Selected papers on confocal microscopy. Milestone Series MS 131. Bellingham, WA: SPIE Optical Engineering Press.

Masters BR. 2000. The scientific life of Maria Göppert-Mayer. Optics and photonics news, vol. 11. Washington, DC: Optical Society of Americ. p 38-41.

Masters BR. 2003. Selected papers on multiphoton excitation microscopy, Milestone Series MS 175. Bellingham, WA: SPIE Optical Engineering Press.

Masters BR, So PTC, Gratton E. 1997. Multiphoton excitation fluorescence microscopy and spectroscopy of in vivo human skin. Biophysical J 72:2405-2412.

Masters BR, So PTC. 1999. Multi-photon excitation microscopy and confocal microscopy imaging of in vivo human skin: a comparison. Microsc Microanal 5:282-289.

Masters BR, So PTC, Kim KH, Buehler C, Gratton E. 1999. Multiphoton excitation microscopy, confocal microscopy, and spectroscopy of living cells and tissues; functional metabolic imaging of human skin in vivo. In: Conn PM, editor. Methods in enzymology, vol. 307. Confocal microscopy. San Diego: Academic Press. p 513-536.

Masters BR, So TPC. 2004. Handbook of biological multiphoton excitation microscopy and other nonlinear microscopies. New York: Oxford University Press.

McClain WM. 1971. Excited state symmetry assignment through polarized two-photon absorption studies in fluids. J Chem Phys 55:2789.

McClain WM, Harris RA. 1977. In: Lim EC, editor. Excited states. New York: Academic Press. p 1-56.

McGrayne SB. 1996. Nobel prize women in science: their lives, struggles, and momentous discoveries. New York: Birch Lane Press. p 175-200.

Peebles PJE. 1992. Quantum mechanics. Princeton: Princeton University Press. p 259-322.
Piston DW, Masters BR, Webb WW. 1995.. Three-dimensionally resolved $\mathrm{NAD}(\mathrm{P}) \mathrm{H}$ cellular metabolic redox imaging of the in situ cornea with two-photon excitation laser scanning microscopy. J Microsc 178:20-27.

Rentzepis PM, Mitschele CJ, Saxman AC. 1970. Measurement of ultrashort laser pulses by three-photon fluorescence. Appl Phys Lett 17:122-124.

Salin F, Squire J, Mourou G, Vaillantcourt G. 1991. Multikilohertz $\mathrm{Ti}: \mathrm{Al}_{2} \mathrm{O}_{3}$ amplifier for high-power femtosecond pulses. Opt Lett 16:1964-1966.

Schäfer FP, Müller H. 1971. Tunable dye ring-laser. Opt Commun 2:407-409.

Shen YR. 1984. The principles of nonlinear optics. New York: John Wiley \& Sons.

Sheppard CJR, Gu M. 1990. Image formation in two-photon fluorescence microscopy. Optik 86:104-106.

Sheppard CJR, Kompfner R, Gannaway J, Walsh D. 1977. The scanning harmonic optical microscope, vol. QE13. Presented at IEEE/ OSA Conf Laser Eng Appl. Washington, DC. p 100D.

Sheppard CJR, Kompfner R. 1978. Resonant scanning optical microscope. Appl Opt 17:2879-2882.

Siegman AE. 1986. Lasers. Mill Valley, CA: University Science Books. p 1004-1040.

Singh S, Bradley LT. 1964. Three-photon absorption in naphthalene crystals by laser excitation. Phys Rev Lett 12: 612-614.

Spence DE, Kean PN, Sibbert W. 1991. 60-fsec pulse generation from a self-mode-locked Ti:sapphire laser. Opt Lett 16:42-44.

Squirrell JM, Wokosin DL, White JG, Bavister BD. 1999. Long-term two-photon fluorescence imaging of mammalian embryos without compromising viability. Nature Biotechnol 17:763-767.

Summers RG, Piston DW, Harris KM, Morrill JB. 1996. The orientation of first cleavage in the sea urchin embryo, Lytechinus variegatus, does not specify the axes of bilateral symmetry. Dev Biol 175 177-183.

Trachtenberg JT, Chen BE, Knott GW, Feng G, Sanes JR, Welker E, Svoboda K. 2002. Long-term in vivo imaging of experience-dependent synaptic plasticity in adult cortex. Nature 420:788-794.

Valdemanis JA, Fork RL. 1986. Design considerations for a femtosecond pulse laser: balancing self phase modulation, group velocity dispersion, saturable absorption, and saturable gain. IEEE J Quant Electron QE-22:112-118.

Van der Waerden BL. 1967. Sources of quantum mechanics. New York: Dover Publications.

Wilson, T. 1990. Confocal microscopy. London: Academic Press.

Wilson T, Sheppard C. 1984. Nonlinear scanning microscopy. In: Theory and practice of scanning optical microscopy. London: Academic Press. p 196-209.

Woodbury EJ, Ng WK. 1962. Proc IRE 50:2367. 